\title{
ANTHROPOS? MENSCHHEIT? MENSCHENZEIT? Medienwissenschaftliche Perspektiven zwischen Skalen, Relationen und inhumanen (Erd-)Geschichten
}

\author{
von FELIX HÜTTEMANN
}

Hannes Bajohr (Hg.): Der Anthropos im Anthropozän.

Die Wiederkebr des Menschen im Moment seiner vermeintlich endgültigen Verabschiedung, Berlin, Boston (De Gruyter) 2020

Eva Horn, Hannes Bergthaller: Anthropozän zur

Einfübrung, Hamburg (Junius) 2019

Frédéric Neyrat: The Unconstructable Earth. An Ecology of Separation, New York (Fordham University Press) 2019

Kathryn Yusoff: A Billion Black Anthropocenes or None, Minneapolis (University of Minnesota Press) 2018

Eine Grenze zu definieren, ist mitunter problematisch, vor allem wenn unklar ist, was dahinterliegt, wer oder was also ausgeschlossen wird. Welche Schwierigkeiten müssen erst entstehen, wenn ein ganzes Erdzeitalter für beendet erklärt und ein neues ausgerufen wird? Dies gilt besonders, wenn sich «der Mensch im betreffenden Zeitalter, dem Anthropozän, befindet und sich als Kollektivsubjekt «Menschheit`selbst zum Grund der Grenzziehung macht.

Die Rahmung Anthropozän birgt unter vorgeblich anderem oder neuem Vorzeichen nochmals die großen Fragen: Was ist Natur? Was ist der Mensch? Was ist Kultur? Was ist Technik?

Die Zäsur proklamiert nicht nur eine Begriffsbestimmung des Menschen als geologischer Faktor, sondern hat normative, epistemische und politische Konsequenzen.
Hinter dem Schlagwort Anthropozän verstecken sich neben posthumanistischen Theorieausprägungen recht unterschiedliche Überbauten und fragwürdige Ideologien.

Allen hier besprochenen Texten stellt sich das Problem, dass sie sich zum Spannungsverhältnis aus ‘Anthropos) als Ursache und Ergebnis verhalten müssen. Die Menschheit als planetarische Kraft steht zum einen als konstituierendes Subjekt, das diese Erdzeit hervorgebracht hat, zur Diskussion. Zum anderen zeigt sich der Mensch im Anthropozän durch seine von ihm bedingte Umgebung als konstituiertes (Gefüge-)Objekt. So muss eine Positionsbestimmung innerhalb der ‘AnthropozänLiteraturs deutlich machen: Anthropos bedeutet Gegenstand und Beobachter_in. Eine Grundproblematik dabei ist: ‘Der` Mensch muss sich fragen, wer spricht, wenn er_sie ‘Anthropos` sagt, und welche Aus- und EinschlieBungen damit vorgenommen werden. ${ }^{1}$

Es scheint, als ob das von Foucault verabschiedete Gesicht am sandigen Meeresufer wiederkehrt. Das mag auf den ersten Blick nicht neu sein, schreitet doch der Pferdefuß der Anthropologie schon seit einiger Zeit wieder durch die Theoriediskussionen. Doch die Herausforderungen des «Anthropos im Anthropozän» - wie der Titel eines der hier besprochenen Bände lautet - liegen darin, «den Menschen/die Menschheit) als Skalierungsbegriff zu hinterfragen und zu diversifizieren. Etwa um die Rolle der humanities in diesem Diskurs zu suchen, den Anthropos im Anthropozän einer negativ-anthropologischen Begriffsbestimmung zu unterziehen oder 
den hegemonialen weiß-männlichen Wissenschaftstyp des Anthropozäns selbst zu kritisieren. ${ }^{2}$ Diesen Ansatz teilen die hier vorgestellten Bücher, denen es zunächst darum geht, «den Menschen` als Subjekt wie Objekt des Diskurses nicht unhinterfragt gelten zu lassen. Es zeigen sich dann weitreichendere politische und wissenschaftliche Problematiken, die in den Texten unterschiedlich thematisch werden. Als erster Problemkomplex erweist sich, dass unter dem Etikett des Anthropozäns und im Fahrwasser des «Menschen als planetarische[n] Akteur[s]» ${ }^{3}$ neben post-humanistischen Ansätzen auch Ausprägungen von ‘Neohumanismen`, «neuen Anthropozentriken und ‘alten` philosophischen Anthropologien zu finden sind.

\section{Ecology of separation}

Frédéric Neyrats The Unconstructable Earth. An Ecology of Separation (2019) widmet sich der neoliberalen Umgebungsveränderung durch Extraktion von ‘Rohstoffen` sowie einer vermeintlich konträren Ökologie der Allverbundenheit oder Ganzheitlichkeit. Der Text zeigt auf, dass sich hinter Schlagworten wie Geo- oder Eco-constructivism, Terraforming oder Geoengineering neoliberale Konstrukte verbergen, denen es um die Beschleunigung anthropogener Umgebungsveränderung und Wertschöpfung geht. Der hypermodernen Austreibung technogener Umgebungen durch noch intensivere technologische Umgebungen setzt Neyrat eine Ökologie der Trennung entgegen. Ähnlich Haraways «everything is connected to something» zeichnet sich die Position Neyrats durch eine poststrukturalistisch beeinflusste Kritik an einer Ökologie der Ganzheitlichkeit aus. ${ }^{4}$

Der Text stellt sich zum einen die Aufgabe, die Konstatierungen hypermoderner neoliberaler Erdveränderungen unter dem Konterfei des Anthropozäns einer Kritik zu unterziehen. Zum anderen ist Neyrat bestrebt, die vermeintlich «nicht-moderne> Gaia-Perspektive und politische Ökologie Latours zu hinterfragen, wobei der Naturbegriff nicht essentialisiert und keine animistische Allverbundenheit der Dinge unterstellt werden soll. Mit seiner ecology of separation schreibt Neyrat gegen die neoliberale Extraktionsideologie und die Idee an, im Zeitalter des Anthropozäns anthropogene Relationen zum Primat zu erheben. Die Pointe des Buches kann darauf zugespitzt werden, dass sich in der Theoriebildung des Anthropozäns der Diskurs zu einem
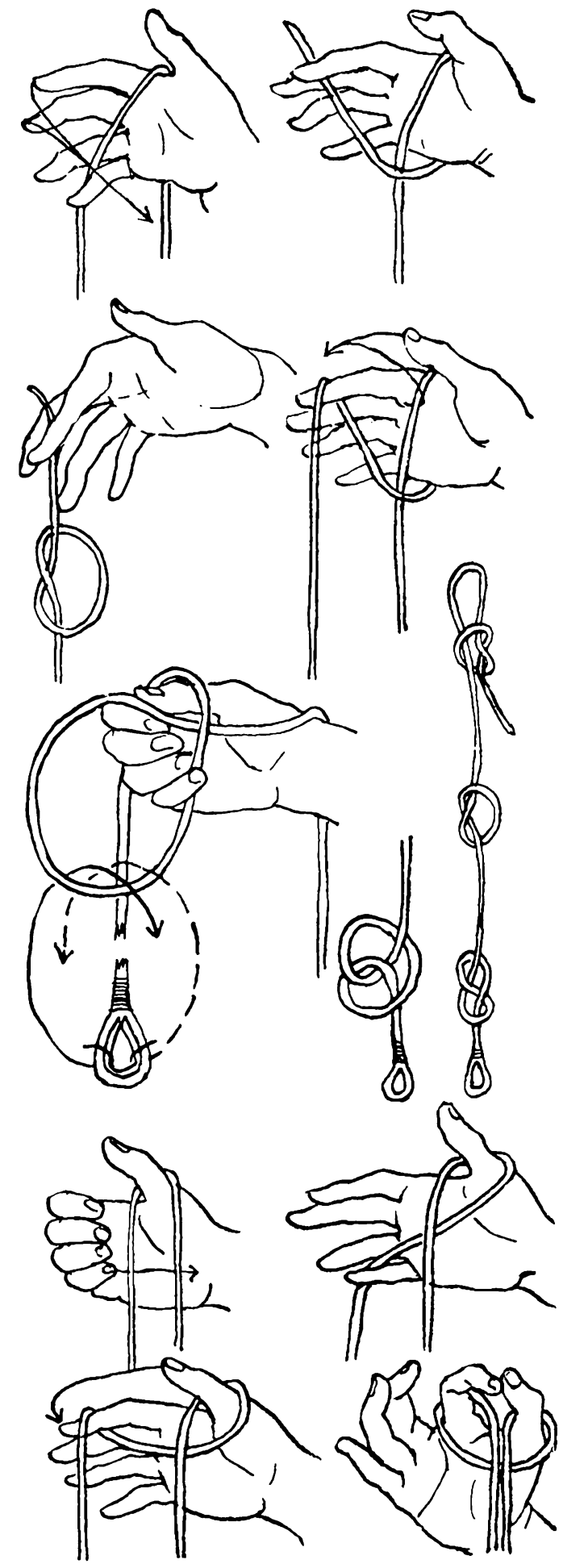

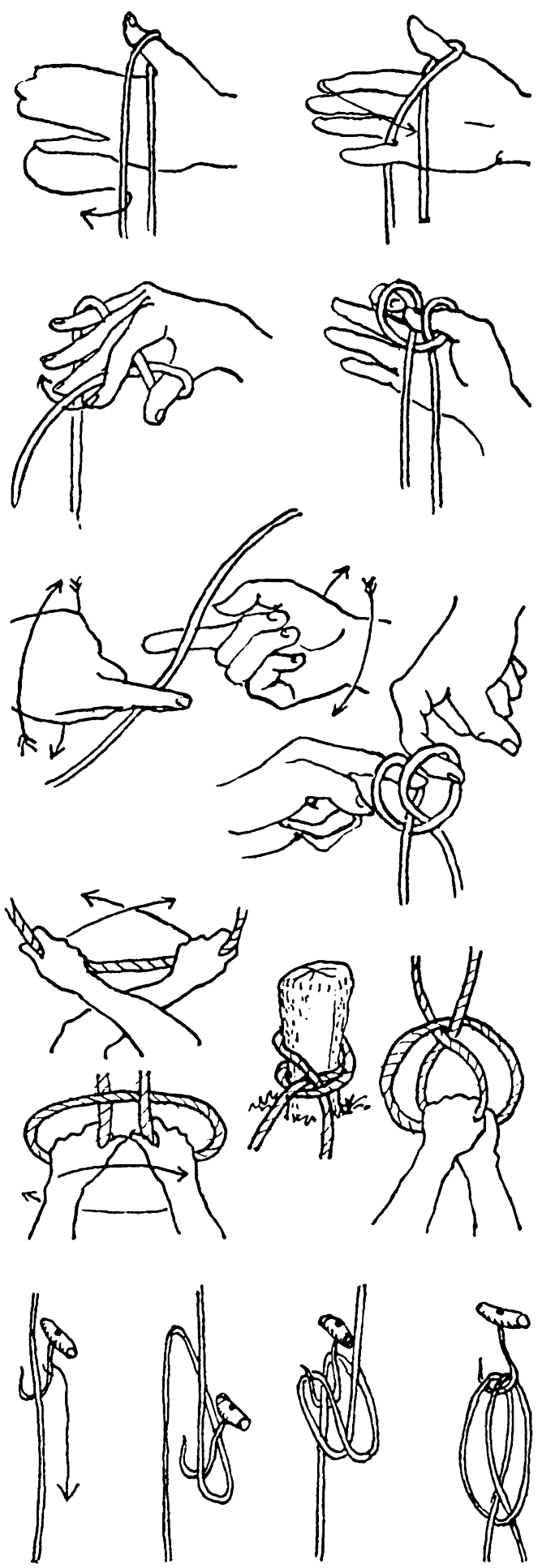

Öko-Konstruktivismus menschgemachter Umgebungsveränderung verschmälere. Denn die Behauptung der Allverbundenheit von menschlicher und nicht-menschlicher Agency unter dem Topos des Anthropos führe dazu, die Agency der non-humans desto stärker in den Hintergrund zu rücken, je mehr auf die Relation von humanem Aktant und environment abgehoben werde. Aus diesem Grund votiert Neyrat für die titelgebende Ökologie der Trennung, welche die Agency der non-humans im Anthropozän ernst zu nehmen versucht und für eine Distanznahme innerhalb sozioökonomischer Situationen plädiert, welche politische Entscheidungen erst möglich mache.

\section{(Negative) Anthropologie}

Die «Menschheit) als Kollektivsubjekt auf planetarischem Niveau zu konstatieren, heißt auch, sich gleichzeitig in die zu beobachtenden Diskurse einzubeziehen und als Beobachter_in zu adressieren. Das macht die Distanznahme zur Akzentuierung non-humaner Agency wie auch jedweden politischen Handelns problematisch.

Weshalb der «Anthropos im Anthropozän» aber nicht zwangsläufig Anthropozentrismus bedeutet und dass sich eine längere Theoriegeschichte des Anthropozäns ausmachen lässt, verdeutlicht der von Hannes Bajohr herausgegebene Sammelband, der auf einer im Jahr 2019 am Leibniz-Zentrum für Literatur- und Kulturforschung (Zfl) Berlin abgehaltenen Tagung basiert. Er zeigt, dass Anthropologie produktiv sein kann, wenn mit Bezügen etwa auf Helmuth Plessner und vor allem Günther Anders von einer negativen Anthropologie, also von einer «Minimalanthropologie» der Unbestimmtheit und nicht von a priori festgesetzten Wesensmerkmalen des Anthropos ausgegangen wird. ${ }^{5}$ Im ersten Teil versammelt der Band Beiträge, die eine philosophische Anthropologie im Anthropozän zu verorten suchen (S. 19ff.). Dem gegenüber stehen im zweiten Teil Texte zu Begrifflichkeiten und Zusammenhängen von ‘Anthropos', Mensch und der Spezies «Menschheit), die als Problematiken der Biopolitik, der Skalierung oder auch des Planetarischen adressiert werden (S. 97 ff.). Der dritte Teil unternimmt einen ‘Brückenschlag) zwischen der «negativen Anthropologie` (S. 173 ff.) und der Anthropozän-Debatte und schließt mit einem Aufsatz von Dipesh Chakrabarty, der die Frage nach der Zukunft der Geisteswissenschaften im Anthropozän diskutiert. ${ }^{6}$ 


\section{Ästhetik des Anthropozäns}

Die Rolle der Geisteswissenschaft ist auch der zentrale Punkt im Junius-Band Anthropozän (2019) von Eva Horn und Hannes Bergthaller. Er liefert eine begriffsgeschichtliche und diskursive Einführung in die primär aus den Naturwissenschaften, vor allem aus der Geologie, stammende Bestimmung des neuen Erdzeitalters.

Im ersten Abschnitt zu Stratigraphien werden, gleich dem geologischen Erfassen von (Erd-) Schichten, einerseits die Begriffe unter anderem des Anthropozäns, des Kapitalozäns und des Anglozäns erläutert. Andererseits werden mögliche Anfänge diskutiert - sei es der Columbian Exchange, die Industrielle Revolution oder die Great Acceleration. Der zweite Abschnitt zu Metamorphosen widmet sich der Frage, wie das Aufkommen des Anthropozän-Diskurses Konzepte und Themen der humanities aufgreift und verändert, wobei die Problematik der Politik und Ästhetik angesichts der «neuen) Menschheitsbedingung im Vordergrund steht. Im dritten Teil zu Verwerfungen argumentiert der Band, dass das Anthropozän einen Bruch mit etablierten Begrifflichkeiten erfordere, um den Herausforderungen für das (bio-) politische und auch ästhetische Handeln gerecht zu werden. Dies pointieren die Autor_innen mit einer aisthetischen ${ }^{7}$ Akzentuierung: «Eine Ästhetik des Anthropozäns, so unser Vorschlag, muss sich weniger mit der Entfremdung von Mensch und Natur als mit deren fundamentaler Verfremdung auseinandersetzen, einem 'Unheimlich-Werden` von Lebenswelt» (S.125). Der Band liefert einen Beitrag zur Historisierung des Diskurses um das Anthropozän, was ihn weit über seine Bedeutung als Einführung hinaus interessant macht.

Einer der hier angesprochenen Problemkomplexe, der ebenso in den anderen Texten eine wesentliche Rolle spielt, ist die Verschränkung von «Menschheit` (oder des «Mensch-Anthropos`) und geologischen Tiefenzeitphänomenen. Dieses Spannungsverhältnis erweist sich als ein Skalierungsproblem, verstanden als eine Frage der Maßstabssetzung, wie sie sich etwa mit Blick auf die Rohstoffgewinnung stellt. So kann eine lokale Einzelhandlung als eine solche betrachtet noch

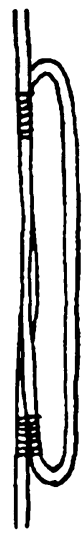

verhältnismäßig unproblematisch sein. Erhebt man diese jedoch zu einem gesamtgesellschaftlichen oder gar globalen Faktor, sieht die Sache wiederum ganz anders aus. Eine Handlung hochoder runterzuskalieren, bedeutet auch, Leerstellen zu verdecken, Spannungen zu überspielen oder gar Marginalisierungen vorzunehmen.

\section{Anthropozän als Extraktions- und Kolonisationsgeschichte}

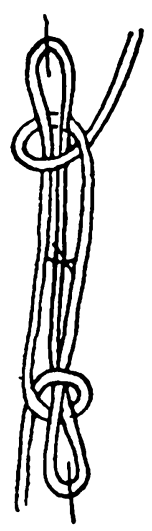

Kathryn Yusoffs A Billion Black Anthropocences or None (2018) unterzieht den Diskurs der geologischen Klassifizierung oder Skalierung einer kritischen Revision, um das Anthropozän anders, d.h. eben nicht hegemonial, weiß und universalistisch zu denken. Dazu nimmt sie eine brown queer feminist perspective einer inhuman geology ein, die sich gegen die patriarchalische Geschichte des Anthropozäns stellt. Sie weist nach, wie stark der Anthropos im Anthropozän Ergebnis einer kapitalistischen, kolonialen und rassistischen Geschichte ist, der sie die marginalisierte und unterdrückte Geschichte derjenigen Akteur_innen entgegenstellt, auf deren Rücken Extraktionen in und von Umgebungen vorgenommen wurden und werden ${ }^{8}$ - sei es in den Goldminen des letzten oder den Coltanminen dieses Jahrhunderts: «Slavery is not a by-product of this process, rather, slavery is driven by an indifferent extractive geo-logic that is motivated by the desire for inhuman properties.» (S. 16) Das Anthropozän, so Yusoffs Schluss, ist immer auch Kapitalozän, das rentmenschlichte) (inhuman) Subjektivitäten produziert. ${ }^{9}$ Sie zeigt damit auf, wie sehr die Diskussion um das Anthropozän eine Fortsetzung kolonialer Dispositive ist.

Yusoffs Text leistet eine Form von Wissensproduktion, die die Leser_innen darauf aufmerksam macht, dass etwa golden spikes zu setzen immer ideologisch ist. Golden spikes bezeichnen die Markierungen von Anfangspunkten solcher Erdzeitalter und Umbrüche. Das ıgolden-spikeSetzen) ist im Grunde eine wissenschaftspolitische Praxis der Evidenz-Erzeugung, gegen die Yusoff argumentiert. A Billion Black Anthropocenes or None stellt damit zum einen die kolonialen 
Inskriptionen in den (Wissenschafts-)Diskurs heraus. Zum anderen elaboriert die Studie Fragen von Materialität, Zirkulation, Extraktion und world-building jenseits hegemonialer Wissenschaft, wie exemplarisch für die Geologie aufgezeigt wird. Diese Revision ließe sich für die Auseinandersetzung der humanities mit dem Anthropozän fortsetzen bzw. sollte fortgesetzt und übertragen werden: «The organization and categorization of matter enact racialization.» (S. 82)

\section{Ein, zwei, viele Anthropozän?}

So verschieden die einzelnen Stränge der Bücher sind, so eint sie die Auseinandersetzung mit dem Anthropos, der Skalierung und Extraktion sowie mit neoliberalen bzw. kapitalistischen Umgebungsveränderungen. In der Betrachtung des Diskurses an den hier exemplarisch ausgewählten Büchern wird deutlich, dass eine kritische Position der humanities angesichts des «Menschen als planetarische[n] Akteur[s]" ausgearbeitet werden muss. So kann die Rolle einer Medienkulturwissenschaft darin bestehen, ihre Tradition eines post- oder auch antihumanistischen Zugangs vielleicht nicht einfach nur zu reaktualisieren, sondern auch eine Auseinandersetzung mit der notwendigen Kritik an einer hegemonialen Variante der Wissensproduktion unter dem Lemma des Anthropozäns anzustoßen, welche bedeuten könnte, sich nicht so, sondern anders, nämlich heterogen, prozessual und relational zu konstituieren.
1 Vgl. Dipesh Chakrabarty: The Climate of History: Four Theses, in: Critical Inquiry, Bd. 35, Nr. 2, 2009, 197-222.

2 Vgl. Jennifer Gabrys u. a.: Dinge anders machen. Feministische Anthropozän-Kritik, Dekolonisierung der Geologie und «sensing» in Medien-Umwelten, in: Zeitschrift für Medienwissenschaft, Bd. 12, Nr. 23: Zirkulation, 2020, 138-151. 3 Hannes Bajohr: Keine Quallen. Anthropozän und negative Anthropologie, in: ders.: Der Anthropos im Anthropozän. Die Wiederkehr des Menschen im Moment seiner vermeintlich endgültigen Verabschiedung, Berlin, Boston 2020, 1-16, hier 2.

\section{4 «Nothing is connected to} everything; everything is connected to something.» Donna Haraway: Staying with the Trouble. Making Kin in the Chthulucene, London 2016, 31.

5 Vgl. Günther Anders: Die Weltfremdheit des Menschen. Schriften zur philosophischen Anthropologie, München 2018; Helmuth Plessner: Die Stufen des Organischen und der Mensch. Einleitung in die philosophische Anthropologie, Berlin, New York 1975. Vgl. im besprochenen Band Bajohr: Keine Quallen; vgl. auch Christian Dries, MarieHelen Hägele: Die Stellung des Menschen im Anthropozän: Ein Brückenschlag zwischen Posthumanismus und Philosophischer Anthropologie, in: Bajohr (Hg.): Der Anthropos im Anthropozän, 173-190.

6 Vgl. Dipesh Chakrabarty: Die Zukunft der Geisteswissenschaften im Zeitalter des Menschen: Eine Notiz, in: Bajohr: Der Anthropos im Anthropozän, 233-237.

7 Darunter verstehe ich, nach der Bedingung der Möglichkeit von Wahrnehmung im Hinblick humaner und non-humaner Agency zu fragen.

8 Vgl. auch Jussi Parikka: A Geology of Media, Minneapolis 2015.

9 Vgl. zur Produktion von Subjektivität im Kapitalismus Gérard Granel: Die totale Produktion. Technik, Kapital und die Logik der Unendlichkeit, Wien 2020.
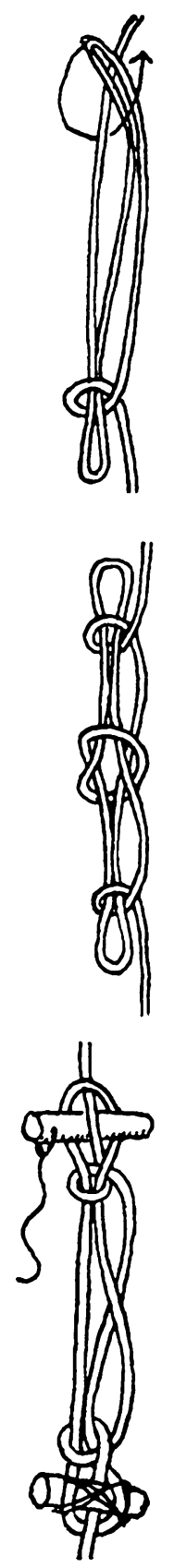